\title{
Herpes Zoster Infection in Patient with Chronic Myeloid Leukemia on Imatinib
}

\author{
Sarah A Elkourashy and Mohamed A Yassin* \\ Department of Medical Oncology, National Center for Cancer Care \& Research, \\ Qatar
}

*Corresponding author: Mohamed A Yassin, Consultant Hematologist, Assistant

\section{Case Report}

Volume 1 Issue 2

Received Date: September 04, 2017

Published Date: October 03, 2017

DOI: $10.23880 / \mathrm{hij}-16000105$

Professor of Clinical Medicine, Weill Cornell Medical College in Qatar, Qatar, E-mail: Yassin@hamad.qa

\section{Abstract}

Varicella-zoster virus is a member of the Herpesviridae family. It can cause chickenpox and Herpes zoster infection (shingles). The latter is an acute, cutaneous infection caused by reactivation of a primary varicella infection upon decline in the virus-specific and cell-mediated immunity. Herpes zoster Infection is a serious infection in immune compromised patients. Predisposing factors include long steroid use, immunosuppressive medications, HIV, solid and hematological malignancies, hematopoietic stem cell transplant, exposure to radiotherapy, infections, \&other forms of stress. Here we report one case of Herpes zoster infection in patient with Chronic Myeloid Leukemia, chronic phase, after four years of Imatinib mesylate, in which complete cytogenetic and molecular remission achieved. This is an exceptional rare complication of Imatinib in Chronic Myeloid Leukemia patients without stem cell transplantation.

\section{Case Report}

Thirty-two year old Nepalese male patient known case of chronic myeloid leukemia (CML), chronic phase, since 2013, on Imatinib $400 \mathrm{mg}$ once daily as upfront therapy in complete hematological remission (CHR), complete cytogenetic remission (CCyR) \& major molecular response (MMR4), followed as per European Leukemia Net recommendations 2013, presented to Hematology outpatient clinic with three day history of acute severe burning pain localized to the left abdominal wall and lower chest. Examination showed grouped tender herpetic form vesicles on an erythematous base running in dermatomal distribution on the left half of the lower thoracic wall as shown in Figure $1 \& 2$.

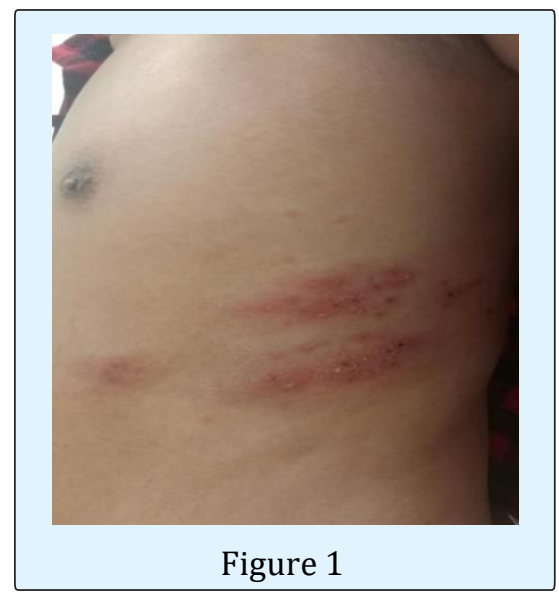




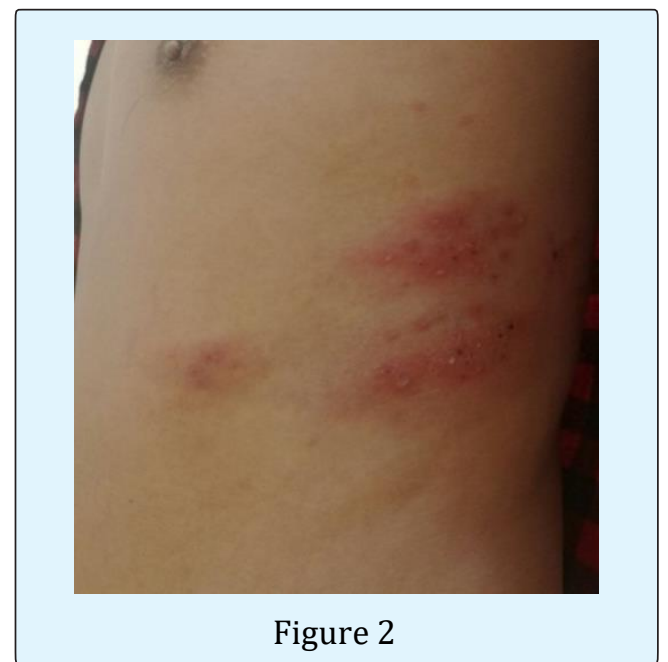

Figure 1\&2 representing the skin rash up on presentation which sounds typical of Herpes zoster infection.

\section{Discussion}

Chronic myeloid leukemia is one of the myeloproliferative neoplasms, as per World Health Organization 2016 classification for hematopoietic and Lymphoid tissues, and it is a triphasic disease which can present as chronic, accelerated or blast phase [1-3]. Tyrosine kinase inhibitors revolutionized the natural course of the disease [4,5]. Imatinib Mesylate was the first generation tyrosine kinase inhibitor to be used for treatment of CML with good tolerability, efficacy, and high safety profiles [4,5]. However, many emerging complications and associations were described with Imatinib. One of extremely rare complications reported with Imatinibis Varicella-Zoster Virus (VZV) infection in CML patients without stem cell transplantation (SCT) [3].

One major retrospective study conducted for CML in the Departments of Leukemia and Bioimmunotherapy at The University of Texas M.D. Anderson Cancer Center; under the name "Development of VaricellaZoster Virus Infection in Patients with Chronic Myelogenous Leukemia Treated with Imatinib Mesylate" by Mattiuzzi GN, et al. [3] 2003, in which 771 patients with CML treated with imatinib Mesylate were included [3]. Sixteen patients (2\%) developed a VZV infection [15 episodes of herpes zoster (HZ), 1 varicella]. The baseline characteristics of the 16 patients with infection do not differ significantly from those who did not develop VZV infection, except for time from diagnosis of CML to imatinib (median: 55 versus 25 months, $\mathrm{P}=0.0056$ ) and the number of prior therapies (3 versus $1, P<0.001$ ). All patients treated with antiviral agents with good response [3].
This study concluded that that imatinib therapy in CML patients not undergoing intensive cytotoxic chemotherapy or SCT is associated with low incidence of $\mathrm{HZ}$ infection [3]. VZV infection is more frequent with longer duration of CML disease, with prior therapies, does not disseminate, responds well to therapy, and does not mandate a recommendation for $\mathrm{HZ}$ prophylaxis in such patients [3]. These characteristics were met in our patient except that Imatinib was used as upfront treatment. Reactivation of latent viral infection appears to be the mechanism of VZV infection due to absence of nosocomial transmission. After primary infection, the VZV becomes dormant in the cerebral or posterior root ganglia and may reactivate, particularly in the presence of defects in cell-mediated immunity. Normal T-cell proliferation is suppressed by Imatinib in a dose-dependent fashion [6]. Imatinib causes suppression of cytokine synthesis by the activated CD4+ $\mathrm{T}$ cells in CML patients [6]. It is important to mention that Interferon-alpha may be protective against herpes viral infections [7] and Hydroxyurea, commonly used for treatment of CML, may also exhibit antiviral properties [8]. This explains low incidence of VZV infection in CML patients receiving any of those treatments.

\section{Conclusion}

Varicella-zoster virus infection is reported rarely in CML patients on Imatinib. It was more observed with longer duration of the disease. It responds well to antiviral treatment and does not require further prophylaxis. It would be important to continue follow up of these patients who will be on long term treatment with Imatinib and be vigilant for the occurrence of such complications.

\section{References}

1. Arber DA, Orazi A, Hasserjian R, Thiele J, Borowitz MJ, et al. (2016) The 2016 revision to the World Health Organization classification of myeloid neoplasms and acute leukemia. Blood 127(20): 2391-2405.

2. Baccarani M, Deininger MW, Rosti G, Hochhaus A, Soverini S, et al. (2013) European LeukemiaNet recommendations for the management of chronic myeloid leukemia: 2013. Blood 122(6): 872-884.

3. Mattiuzzi GN, Cortes JE, Talpaz M, Reuben J, Rios MB, et al. (2003) Development of Varicella-Zoster Virus Infection in Patients with Chronic Myelogenous Leukemia Treated with Imatinib Mesylate. Clin Cancer Res 9(3): 976-980. 
4. O'Brien SG, Guilhot F, Larson RA, Gathmann I, Baccarani M, et al. (2003) Imatinib Compared with Interferon and Low-Dose Cytarabine for Newly Diagnosed Chronic-Phase Chronic Myeloid Leukemia". N Engl J Med 384(11): 994-1004.

5. Sacha $\mathrm{T}$ (2014) Imatinib in Chronic Myeloid Leukemia: an Overview. Mediterr J Hematol Infect Dis 6(1): e2014007.

6. Gao H, Lee BN, Talpaz M, Donato NJ, Cortes JE, et al. (2005) Imatinib mesylate suppresses cytokine synthesis by activated CD4 $\mathrm{T}$ cells of patients with chronic myelogenous leukemia. Leukemia 19(11): 1905-1911.

7. Jonasch E, Haluska FG (2001) Interferon in Oncological Practice: Review of Interferon Biology, Clinical Applications, and Toxicities. The Oncologist 6(1): 34-55.

8. Neyts J, De Clercq E (1999) Hydroxyurea Potentiates the Antiherpesvirus Activities of Purine and Pyrimidine Nucleoside and Nucleoside Phosphonate Analogs. Antimicrobial Agents and Chemotherapy 43(12): 2885-2892. 